\title{
Hybrid intelligence aspects of programming in *AIDA algorithmic pictures
}

\author{
Yutaka Watanobe $^{\mathrm{a}, *}$, Nikolay Mirenkov ${ }^{\mathrm{a}}$ \\ ${ }^{a}$ Graduate Department of Information Systems, University of Aizu, Aizu-Wakamatsu, \\ Fukushima 965-8580, Japan
}

\begin{abstract}
Programming in algorithmic pictures ( $\boldsymbol{a}$-pictures) is an approach where pictures and moving pictures are used as super-characters for representing features of computational algorithms and data structures. Within this approach some "data space structures" are traversed by "fronts of computation" and/or some "units of activity" are traversed by flows of data. There are compound $\boldsymbol{a}$-pictures to define algorithmic steps (called Algorithmic CyberFrames) and generic $\boldsymbol{a}$ pictures to define the contents of compound pictures. Compound $\boldsymbol{a}$-pictures are assembled into special series to represent some algorithmic features. The series are assembled into an Algorithmic CyberFilm. The generic/compound $\boldsymbol{a}$-pictures and their series are developed and acquired in special galleries of an open type where supportive pictures of embedded clarity annotations are also included. In this paper, *AIDA (Star-AIDA) modeling/programming language (AIDA stands for Animation and Images to Develop Algorithms) and its Filmification modeling (F-modeling) environment are briefly considered and examples of programs in $\boldsymbol{a}$-pictures are provided. A special attention is paid to *AIDA programs as special information resources which perception, comprehension and cognition depend on interaction with, at least, a few different but mutually supplementing features of $\boldsymbol{a}$-pictures. A scheme of data/knowledge acquisition based on clusters of different views and how this acquisition is orien-
\end{abstract}

*Corresponding author. Tel.: +81-242-37-2715; fax: +81-242-37-2753

Email address: yutaka@u-aizu.ac.jp (Yutaka Watanobe)

Preprint submitted to Elsevier

September 27, 2013 
ted to enhancing user's ability within works on developing application models, corresponding algorithms and programs are presented.

Keywords: Programming in pictures, *AIDA, Algorithmic CyberFilm

\section{Introduction}

The natural intelligence is related, in a great part, with people abilities to perform mental simulation, including thinking/reasoning, problem solving, and decision making [13-14][18]. However, this simulation depends not only on internal information processing mechanisms of the brain, but also on levels of abstraction connecting the physical world things and corresponding abstract models. Perception and cognition of people are usually much higher if they perform mental manipulations with real objects, and essentially decreased if abstract information objects are involved. Syntax-semantics gap is a fundamental basis for many problems related to the perception and cognition. This gap is predefined by existing systems of symbols, notations, languages, as well as by ambiguous terminologies, trans-disciplinary and cross-cultural contexts. It also depends on the representation of dynamical processes of modeling problems and the adaptive mechanisms of working systems. To bridge the syntax-semantics gap and decrease burden of mental simulation, modern information systems are supported by special data/knowledge acquisitions, ontologies and high-level interfaces based on different mental strategies related to different decision functions (see, for example, [11][29]). These supportive approaches facilitate knowledge sharing and reuse, and are based on a machine-processable semantics of information resources which can be provided to different agents. Though such agents are promoted to be both software systems and humans, in fact, the latter have serious problems with understanding and use of "formal specifications of a shared conceptualizations." In general, to enhance people ability in understanding meaning of objects and processes and in performing mental simulation, there is a necessity not only to increase the levels of symbols, notations and languages, but also to update our current concept of abstraction. Now abstraction is eva- 
luated as a number of attributes/parameters which can be ignored (covered) for simulating some objects or processes. Instead, it should be evaluated by a number of attributes/parameters which should be preserved in order people can still understand the corresponding model meaning. The current technologies, including processor performance and memory sizes, allow us to take steps for such updating.

The intelligence of software for computational engineering, as for other computational fields, is usually based on a collection of decision support technologies for application researchers and practitioners in creating reliable models and corresponding programs, and in performing relevant experiments and simulations. The aims of the technologies are to enable people for making faster and better decisions, as well as for producing robust solutions. Such technologies are embedded in languages of specification and modeling, in special supportive tools and unified environments, as well as in some systems of data/knowledge acquisition. Roughly, these languages, tools, and systems can be divided to two subsets: one with orientation to make decisions instead of users and another with orientation to help users in making their own decisions (see, for example, [2][5-6][10][36]). In fact, a majority of the technologies include features of both types with some prevalence of one of them.

Our motivation is to develop a modeling/programming environment where users can feel themselves more comfortable in developing new models and corresponding algorithms, and in representing them in forms recognizable and understandable by other users. In this environment, the confidence of users in making decisions is based on access to previous decisions of others, on quick perception and understanding these decisions and on their transformations leading to new models and algorithms. To reach such a goal, special methods for data/knowledge acquisition are implemented. They are based on clusters of different views representing hybrid forms of information resource features. Additional aspects of our motivation are to create such hybrid forms which can make our modeling/programming environment more competitive in expressing details of application algorithms and which can use animation for presenting 
behavioral features of model constructs.

In this paper we briefly describe *AIDA modeling/programming language employing pictures and moving pictures as algorithmic super-characters ( $\boldsymbol{a}$ characters) for representing features of computational algorithms. Special attention is paid to intelligent aspects of *AIDA and its environment with a focus on data/knowledge acquisitions which have influence on user's perception, comprehension, and cognition abilities. We also present a number of techniques which are used with programming in $\boldsymbol{a}$-pictures for enhancing the abilities of people working on developing application models and corresponding algorithms. These techniques are based on acquiring new super-characters, their compositions within compound constructs and template programs involved, on applying background, foreground and auxiliary images, and on representing scalable forms of model spaces and attributes declarations, dynamical features (animation) of algorithmic steps, and multiple views of an algorithm as a whole.

\section{Related works}

There is a great variety of works which have some relation and influence on our research activity. However, here we have an opportunity to mention only a selected part of them. First of all, we would like to point new tools related to developing human-computer interfaces for the Internet applications [4], and environments where the concept of putting human needs first is applied and where a necessity for uniting scientific and artistic sides, to obtain a balance between usefulness and attractiveness of technologies, are considered [31].

We would also like to point a paper related to cognitive aspects of software design [9], where authors try to find solutions by considering answers to the following questions: is it possible to bring together theoretically a computer program and a natural language text, and what are differences between programming languages and natural languages. Other papers of our interests are [16][25], where cognitive and computational features of diagrams are considered through another question: why a diagram is (sometimes) worth ten thousand 
words. The authors point that the fundamental difference between diagrammatic and sentential representations is that the diagrammatic representation preserves explicitly the information about the topological and geometrical relations among the component of the problem, while the sentential representation does not. In addition, we would like to mention approaches related to analogical representations of programs (including a semiotic analysis of comics) [12], self-assembling tilings (for the creation of the desired shapes) [1], various types of information resources annotations [23-24], and the long-term activity of researchers in Certec (Sweden) [8] (where the use of pictures as language for people with disabilities is promoted).

Special points of our attention are ways supporting the application programmers in their decisions by enhancing their perception, cognition, and comprehension abilities. This is abilities to integrate, analyze, and act on large amounts of data from various sources at once [22]. Existing programming environments based on UML (including Executable UML [15][19][28]) and other diagrams are still not easy with abstract, necessity to possess specific skills for putting together different views, limited number of options and with understanding dynamical processes through static structures [3][26-27]. In addition, they are not good for detailed programming and textual action languages with appropriate semantics are usually involved. A great part of the programming work should be done in such languages (Alf or just $\mathrm{C}$, Java, $\mathrm{C}++$, etc.). Then what do we really abstracted away and what is difference between programming and modeling? [21]. In addition, they are not so good in displaying "large amounts of data" to be useful for enhancing the above mentioned perception, cognition, and comprehension abilities. Another important thing for us is the software intelligence and forms of data/knowledge acquisitions within the corresponding environments and tools [17]. In conventional programming environment such acquisitions are, in a great part, reduced to adding new items in libraries of components, classes, and/or procedures. These items of black-box types are useful in general, but not so supportive in enhancing "see, think and quickly understand" abilities of the users. 


\section{Programming in $a$-pictures: a brief overview}

As we have mentioned in the introduction, people cognition abilities, in a great part, depend on their abilities to perform mental simulation. However, this simulation depends not only on internal information processing mechanisms of the brain, but also on levels of abstraction connecting the physical world things and corresponding abstract models. To make such simulation more efficient, it is necessary to decrease spending of people energy and time for the recognition of semantics behind unknown terminology, for understanding associations between objects which syntax-semantics forms are based on rote memorization, and for fighting with concepts and notations that have a trend to be over-abstracted. In other words, disorientation and cognitive overload are serious obstacles for people. They become tired after overcoming such obstacles and do not have enough energy and time for focusing on their own ideas and models, and on the use of their real knowledge and cognitive abilities.

Programming in $\boldsymbol{a}$-pictures (we also call it as Filmification of Methods) is a special approach to help people for some saving their energy and time. It is supported by a special environment where pictures and moving pictures (animation) are used as an open set of algorithmic super-characters ( $\boldsymbol{a}$-characters) for more direct representation of features of computational algorithms and data structures. Within this approach an algorithm is considered as "activity" in 4-dimential space/time. It is represented by some "parameterized data-space structure" traversed by "fronts of computation" and by necessary operations performed during this traversal process (it can also be represented by some diagrams with activity unit nodes traversed by flows of data).

There are compound $\boldsymbol{a}$-pictures to define algorithmic steps (called algorithmic CyberFrames) and generic $\boldsymbol{a}$-pictures to define the contents of compound pictures. Compound $\boldsymbol{a}$-pictures are assembled into special series to represent some algorithmic features. A number of the series is assembled into an Algorithmic CyberFilm. $\boldsymbol{A}$-characters represented by algorithmic pictures and/or animation (all are called $\boldsymbol{a}$-pictures) are acquired in special galleries. In 
some cases one $\boldsymbol{a}$-character can be represented by several $\boldsymbol{a}$-pictures (in a part, like one conventional character can be represented by different fonts).

Figure 1 depicts a structure scheme of the Algorithmic CyberFilm format. The top-right series of frames represents Main (Integrated) view of an algorithm. Many $\boldsymbol{a}$-characters involved in the view are intuitively understandable and do not require serious efforts for memorization. However, some of them can be not so intuitively understandable. In addition, different people can have different levels of cognition and perception. To enhance the comprehension of the $\boldsymbol{a}$ characters and their compositions, each picture of the Main view frames is supported by corresponding meaning annotation and/or by a series of frames of the bottom layer views. On the bottom layer there are Algorithmic Dynamics, Command and Interface views. They are also to clarify (if necessary) meaning of corresponding $\boldsymbol{a}$-characters. Series of frames of Dynamics view clarify meaning of CyberScene $\boldsymbol{a}$-characters by presenting animation of CyberScene skeletons (space data structures and traversal schemes of computational flows on these structures). Series of frames of Command view disclose some details related to application values of variables and formulas (actions) that are attached to the space-time points of the skeletons. Series of frames of Interface view represent extended vision of input/output operations that define the algorithmic interface with external world, as well as how a software component based on the algorithm should look from outside.

For each series of frames there is its own set of $\boldsymbol{a}$-characters. As a result, the programming environment for making Algorithmic CyberFilms can be considered as a set of four mutually supplemented languages (and supporting systems) to define four different views of each software component. Within this environment, the application users usually do not create frames, algorithmic skeletons and necessary template programs, but take and assemble them from ontology type libraries. For these users, the programming is reduced to defining sizes of the space structures, declaring variables on these structures and specifying operations on traversal nodes, including input/output operations.

Filmification of methods has been applied to a great variety of algorithms in- 
cluding sequential and parallel matrix multiplications, sparse matrix-vector operations, solving algebraic and partial differential equations, cellular automationlike algorithms, as well as to algorithms on trees, pyramids, particles-in-cells, etc. (see, for example, [20][30][37]). A large set of algorithms on graphs and a corresponding library have been presented in [34-35]. The knowledge and experience accumulated within this work were transformed into *AIDA language and Filmification modeling (F-modeling) environment including *AIDA compiler (a $\mathrm{C}++$ code synthesizer from program in pictures) and a set of editors for different views and annotations [33][38]. In this paper we focus on cognitive and intelligent aspects of programming in algorithmic pictures and how they are supported by F-modeling environment. This focus also includes acquiring clusters of hybrid pieces of knowledge that employ the most appropriate formats bridging the gap between syntax and semantics in algorithm representations.

Colors play a very important role in *AIDA programming, so colored figures used in the paper are available at a web-site [7].

Our results are a first step in rich research activities which can be based on applying hybrid forms of information resources. Multiple views representing a number of features of an object can be used not only for modeling/programming constructs and algorithm specifications, but also for various meta-data explanation, educational material preparations, etc. Remote access to information prepared in "self-explanatory" (multiple views) format is a new way for doing many things on the Internet. The introduction of super-characters (with clusters of features behind) provides a great potential for all types of information resources, but requires some reconsidering human-computer interfaces, syntax/semantics control, data/knowledge acquisition, code generation, etc. In other words, *AIDA language and F-modeling environment can be used as a test-bed for various innovations in IT research and implementations. 


\section{Examples of programs in $a$-pictures}

To illustrate some of the above mentioned features of the Algorithmic $\mathrm{Cy}-$ berFilm technology, let us consider a few examples of programs in $\boldsymbol{a}$-pictures related to adjustments of physical field attributes and a Dirichlet problem for Laplace's equation.

Example 1. There is a physical field represented by an integer attribute on a 2 -D mesh. The task is to find all nodes with negative values of the attribute and replace these values by " 0. "

The Main view of *AIDA program for this example is presented by Figure 2. The first row shows the declaration of a scalable 2-D structure and variable $\mathbf{A}$ (in fact, a 2-D array) which integer elements are associated to all nodes of the structure; a micro-icon (generic $\boldsymbol{a}$-picture) to the left of $\mathbf{A}$ specifies the integer type of the elements.

The second and fourth rows show input and output operations. Each of these rows includes an $\boldsymbol{a}$-picture of a scene, a triple-circle micro-icon (also a generic $\boldsymbol{a}$-picture) of a collective operation, and an expression of the operation. An $\boldsymbol{a}$-picture of a scene, if we look at its Dynamics Details view, usually presents a series of steps where some highlighting (flashing) nodes demonstrate various computational activities on a structure. In our example, the series includes only one step where all nodes of the 2-D structure are flashed by the same red color. The "caption" at the bottom of the $\boldsymbol{a}$-picture requires specifying only one type of computational activity related to this color. Usually, this means that such activity is performed in parallel on all highlighting nodes. However, in our example, a collective activity (operation) is used. A micro-icon of a collective operation is next to the $\boldsymbol{a}$-picture. It means that data, in some indivisibility manner, are taken from all highlighting nodes, processed and returned within the operation which on default knows what and where should be done. In the second row, it is an input operation that reads data from a file and distributes them among elements of $\mathbf{A}$ on the highlighting nodes. In the fourth row, it is an output operation that displays values of $\mathbf{A}$ at monitor through the default 
use of a "value-to-color" function. Some details of this function are available from environment settings or annotations.

The third row defines the basic computation by an $\boldsymbol{a}$-picture of a scene, a micro-icon of an individual operation, and an expression of the operation. The $\boldsymbol{a}$-picture presents a series of steps of a 2-D structure traversal scheme in "topdown, row-to-row, left-to-right" manner. At each step, only one node is flashed. In this node, the operation based on selecting maximum of two arguments is executed.

Example 2. There is a physical field represented by a real attribute on a 2-D mesh but this field has an internal sub-field of "no activity zone." The task is like in the previous example to find all available nodes with negative values of the attribute and replace these values by "0."

In this example (Figure 3), a micro-icon for the declaration of double-real type elements, icons for specifying masks ("no activity zone") and "if-then" constructs, as well as a function generating random number in the $(-5.0,5.0)$ interval are shown. The mask area is defined by values of $(\mathrm{r} 1, \mathrm{c} 1)$ and $(\mathrm{r} 2, \mathrm{c} 2)$ coordinates, and the "if-then" construct is disclosed through "condition" and "operation expression" related to rhombus and wave micro-icons. The colored vertical lanes are used to enhance the visual perception of the condition and operation expressions (especially, for cases when they are rather complex). Numbers on these lanes (as well as under highlighted circles of the traversal scheme activity) are just labels to distinguish colors; this is important for people who are not good in color recognition, etc.

In this example, the mask has a name of obst (obstacle). It can be put in a library and called by the name. In such a case, coordinates (r1,c1) and (r2,c2) can be covered and shown only on demand.

Example 3. Assume we solve a Dirichlet problem for Laplace's equation in the interior of a given 2-D region that takes prescribed values on the boundary of the region. In addition, assume that Jacobi relaxation algorithm is applied.

By this example we explain an observer node structure and corresponding variables, as well as new traversal schemes, computation on boundaries and for- 
mula patterns. In addition, clarity annotations based on Algorithmic Dynamics view are also introduced.

Laplace's equation $\frac{\partial^{2} A}{\partial x^{2}}+\frac{\partial^{2} A}{\partial y^{2}}=0$ on a 2-D mesh of $\mathbf{N} \times \mathbf{M}$ size with some values on the boundary of the domain is solved by the Jacobi relaxation algorithm that is by an iteration process where at each step new values of $\mathbf{A}$ in internal points of the mesh are calculated based on values of the previous step in the neighboring points (defined by a finite difference scheme). Assume that variable $\mathbf{B}$ is used for keeping the new iteration values and variables $\mathbf{e}$ and $\mathbf{I}$ are for checking the process of convergence. Variables $\mathbf{A}, \mathbf{B}$ and $\mathbf{I}$ are attached ("associated") to the 2-D structure specialized on presenting computation distributed in space and $\mathbf{e}$ is attached to a special one-node structure specialized for presenting centralized computation. This node is also called "an observer node" where indivisible operations are performed. These operations can read variable values from space structures, but cannot change them. Only variables associated with the observer node can be changed by the operations.

Figure 4 is a Main view CyberFrame defining the algorithm. The first row in the frame is the declaration of the 2-D structure and one-node structure, as well as variables (including their types: double-real for $\mathbf{A}, \mathbf{B}$, and $\mathbf{e}$; integer for I) associated to the structures. The computation includes two scenes for internal node values input and boundary values initialization, and one scene for a main part of the algorithm and results visualization. Each scene has its own $\boldsymbol{a}$-picture with a "caption" pointing operations which should be defined within the scene computation. The scene $\boldsymbol{a}$-pictures are depicted by the left column of the frame and request (from top-to-bottom) specifying 1, 1, and 4 operations, respectively. The operations are defined by the expressions presented to the right of the $\boldsymbol{a}$-pictures.

The first scene is just a collective operation to input the initial values of A for internal nodes. The second scene is scanning boundary nodes of the 2$\mathrm{D}$ grid and performing in the node of the visit the rightly defined formula (a highlighted node with a vertical/horizontal line means a number of row/column where the node of the visit is located). The third scene is the main part of 
the algorithms. In the expression for the computation of $\mathbf{B}$, short arrow bars around the red (dark-gray) circle point out that values of variable $\mathbf{A}$ are not taken from a node highlighted by the traversal scheme, but from a node of a corresponding neighborhood (of right, top, left, and bottom directions).

For cases where the scene $\boldsymbol{a}$-pictures are not so intuitively understandable, the user can request its semantics at any moment, as shown by Figure 5 and Figure 6. In fact, in these figures, frames of Algorithmic Dynamics view are shown (though a text-based annotation, etc. can be involved). The embedded clarity support is applicable not only to scene $\boldsymbol{a}$-pictures, but also to generic and compound pictures, including micro-icons related to the variable declaration. Figure 7 depicts examples of a physical meaning and units-of-measure clarifications for variable A (representing "Temperature" in Kelvin degrees), as well as unfolded forms of neighborhood where computational activity is performed.

In conventional programming languages, declarations of variables are based on some abstract data constructs where elementary types of "integer, real, double, character, etc." play a fundamental role. Usually, essential efforts should be done to imagine and understand such a construct. For example, to represent a multi-grid (pyramid) construct, a number of 2D-arrays of real or double-real types representing different layers of the pyramid are declared. This declaration does not display a space structure image and does not provide any information about physical meaning of application attributes and units of their measurement. In our programming environment, special images are used as super-characters or as embedded clarity annotations which can be displayed on user's demands. Figure 8(a) depicts a set of super-characters applying for the space structure declarations and Figure 8(b) shows super-characters of traversal schemes on the structures; in addition, Figure 9 depicts a set of supportive images applying for embedded clarity annotations. The supportive images of Figure 9 are composed by a physical attribute name, corresponding unit of measurements, an icon to hint on attribute meaning, etc.

The Figure 8(a) $\boldsymbol{a}$-characters represent some space structures (related to user's model) which nodes and/or edges are used for allocation of application 
attributes (variables). In fact, the attribute elements obtain a "space affiliation." Through such structures the user can see more directly relations between his/her model and variables involved, and visually check these relations. The structures represent scalable spaces which sizes are defined by the users through the assignment of necessary values to $\boldsymbol{a}$-character parameters. For example, the top-left $\boldsymbol{a}$-character in Figure 8(a) is to declare a 3D space structure which size is defined by values assigned to $\mathbf{M}, \mathbf{N}$, and $\mathbf{L}$. In a similar way, the second from the left super-character in the top row is to declare a top-less pyramid space which size is defined by values assigned to $\mathbf{N}$ and $\mathbf{H}$. The attribute elements can be arrays (or other types) and a combined dimension of data involved can be the sum of the array dimension and the space structure dimension. For cases, where the structure spaces can be modified during the computation by applying special operations in appropriate nodes, for example, by cutting operations for removing some tree branches, special versions of $\boldsymbol{a}$-characters are used. These versions are to explicitly point about such features of modeling processes. Special cases of very dynamical processes are related to computation on particles. The bottom-left $\boldsymbol{a}$-character is to represent a 2D-model, where the number of particles involved is related to parameter $\mathbf{N}$.

For majority of the $\boldsymbol{a}$-characters of the above mentioned type, there is a family of images to show "natural" sub-structures of a structure; for example, for a tree structure, leaf nodes or a root node are considered as such sub-structures; for a $2 \mathrm{D}$ structure, the first row and the first column, as well as diagonals are also considered as the "natural" sub-structures. These images are used to declare the variable allocation not on the whole structure, but on its parts. In addition to these images, there are also 1) structure mask images which help in covering some sub-structures from computational activity and introducing various types of irregularities necessary for imitating complex processes of real world, and 2) substructure slices of vertical, horizontal, cyclic and other types which help in specifying inter-component communications and reading/writing access to external data.

The Figure 8(b) $\boldsymbol{a}$-characters represent some "fronts of computations" on 
the structures; details of each of them are explained in the manner of Figure 5-7 and/or with additional annotations.

\section{Background, foreground and auxiliary images}

Algorithmic CyberFilm frames, especially related to dynamical process representations, show some space data structures, traversal schemes on these structures and high-level formulas (activities) on the structure nodes. Corresponding images (visual objects) provide direct influence on the generation of executable codes. Examples of such frames are depicted by the first row of Figure 10. The first frame shows just a graph data structure, the second presents a tree with a node highlighted for some operations, the third shows a grid with four highlighted nodes for identical operations to be performed in parallel and the fourth shows a set of five 1-D structures. Of course, such frames and ideas behind them are understandable much easier in comparison with corresponding representations in the conventional language texts. However, their perception and cognition features will be much higher if additional background images can be involved. Examples of such images are depicted by the second row of Figure 10.

The first frame in this row shows not only a graph data structure, but also a cue that the model considered will be related to Fukushima prefecture territory. The second frame shows that the activity on the tree leaf node has "a history" presented by a trace sub-tree, and the third frame depicts a cue about parallel computation in the past and possible computation in the future. Finally, the fourth frame shows referencing colors behind 1-D structures, numbers in and/or by nodes and some relations arrows. More extended text can also be used for such clarifications. The background images of these frames do not have any influence on the code generation, but are very important for helping people in understanding corresponding semantics and performing mental simulation. Another example of helping images is depicted by Figure 11, where different colors are used to show different sub-graphs involved and edges between them. 
In this case, the helping images and their colors are used not only for clarifying the meaning, but also for defining a half-highlighted operation (that is for the influence on code generation). Frame $\mathrm{X}$ represents that some operations on selecting the node activity at the next frame should be done on a top-right half-highlighted node; for making this selection, data from contour highlighted nodes should be taken. Frame $\mathrm{X}+1$ shows a result of the selection that is a node which was moved from green-color (dark-color) sub-graph into blue-color (light-color) sub-graph and pointed by an arrow.

This frame also shows that some operations should be defined for the topright node based on data from all nodes. In addition to Frame $\mathrm{X}$ and Frame $\mathrm{X}+1$, Figure 11 depicts the definition of the selecting operation mentioned with Frame X. This definition means "assign a counter highlighted node of one subgraph into another sub-graph where an edge between sub-graphs has a minimum value."

In general, the Algorithmic CyberFrame is a multilayer package of images (see Figure 12) including background, main ground and foreground slices. For example, the arrow in Frame $\mathrm{X}+1$ at Figure 11 is represented in this package by a supportive image slice from the foreground layer. Such slices can include conventional text and/or mathematical expressions. The number of slices in the layers depends on semantics to be explained. For many CyberFrames main ground images are only used, but there are also cases of auxiliary frames where only supportive images/texts are involved.

The auxiliary frames help in comprehension of CyberScenes and CyberFilms meaning. They can be considered as cover frames, summary or documentation type annotations. Template programs in $\mathrm{C}++$ implementing CyberScene processes can be called and used as special annotations, too. Basically, there are three types of annotations. The first type is related to algorithmic details views of *AIDA program format and new $\boldsymbol{a}$-pictures. These views and annotations to new $\boldsymbol{a}$-pictures are attached by developers when CyberScenes or CyberFilms and new $\boldsymbol{a}$-pictures are included in libraries and galleries. The second type is related to joint activity of an editor of Main or other views and the user: an editor 
initiates an interaction by asking special questions and the user's answers are transformed into some annotations. Questions about units of measure and/or some features of initial data can be an example of the interactions. Finally, the third type is annotations prepared and attached by the users on their own initiatives. In such cases, balloon type micro-icons are appeared near corresponding $\boldsymbol{a}$-picture-based expressions or constructs.

In other words, *AIDA is a language of explanation of application models, methods and algorithms involved rather than a language of coding. Pictures, animation and conventional texts are used to provide the explanation. Executable codes are generated automatically from such explanations; though only a part of the explanations are applied in the generation.

\section{Current forms of data/knowledge acquisition in F-modeling envi- ronment}

F-modeling environment supports the user activity in creating reliable models and corresponding programs by an open set of very-high level $\boldsymbol{a}$-characters, special constructs combining these $\boldsymbol{a}$-characters to represent algorithmic steps, special assembly lines to define algorithmic scenes, and libraries (in fact, ontologies) to acquire such $\boldsymbol{a}$-characters, constructs and algorithm representations. Pictures and moving pictures (supported by text, if necessary) are applied as expressive forms of these $\boldsymbol{a}$-characters, constructs, and lines. In many cases pictures and animation are much more attractive for understanding an algorithm (and its features) in comparison with conventional programming text. However, there are cases where pictures are still too abstract and animation is too boring. So, our approach is based on multiple views and formats taking into account different knowledge and abilities of users. Within this approach, we follow a cognitive model where success of user's perception, comprehension and cognition depend on interaction with, at least, a few different but mutually supplementing features of an $\boldsymbol{a}$-character, construct, or algorithm. Such multiple views organize a basis for self-explanatory semantics and essentially decrease 
necessity to memorize meaning of $\boldsymbol{a}$-characters which number can be huge. The self-explanatory semantics is supported by specially prepared pieces of knowledge. These pieces employ the most appropriate formats to bridge gap between syntax and semantics.

Permanent acquisition of these pieces of knowledge and best practice behind them allow the adaptation of *AIDA to features of application domains and the enhancement of the language intelligence as a whole. *AIDA and Fmodeling environment are a "living system-organism" where adding appropriate $\boldsymbol{a}$-characters and constructs related to some applications makes the organism this-application-oriented. The acquisition of the pieces of knowledge and the efficient access to these pieces through interaction on a set of interface panels of different view editors are related to one type of the intelligent aspects. Another type is related to checking the *AIDA program correctness.

Each $\boldsymbol{a}$-character has a set of pre-defined positions in compound pictures and each position in a compound picture has a set of $\boldsymbol{a}$-characters allowed on this position. Checking the consistency of such information is performed before analyzing the information of conventional syntax expressions. Essential number of $\boldsymbol{a}$-characters and declared variables, masks, etc. associated with some settings are available on demand. These settings are attached automatically from appropriate libraries (and other acquired information) and/or as a result of special dialog between the user and editors of F-modeling environment. They provide some features of application model, algorithm, initial data, etc. Checking the consistency of different settings and user's answers is also performed before the code generation. All these things essentially prevent the users from making errors. A fundamental part of this prevention is related to checking consistency of multiple sources of different information about the same features of algorithmic CyberScenes. In this checking, there are implicit and explicit sources. Implicit sources are related to multiple views where some overlapping specifications of the model behavior are involved. Implicit sources can also be related to clarity annotations which primary goal is to explain the meaning of some pictures or their composition. Semi-explicit or explicit sources are created by the users as 
a result of their answers to the environment editors.

To demonstrate how the implicit sources can be used for array-bound error discovery, let us consider the following example of Figure 13, where an inconsistency of a traversal scheme on a 2-D structure (presented by the left $\boldsymbol{a}$-picture) with a formula (presented by the right expression) is shown. In this example, the traversal scheme defines column-by-column computation starting at the leftist column, reaching the rightist column and returning back to the leftist column. During this traversal process in each node of the column activated (flashed), the formula is executed. This formula changes value of $\mathbf{A}$ in a node by taking values of $\mathbf{A}$ from two neighboring nodes. This leads to the array-bound errors both at the leftist and at the rightist columns. Such errors are discovered by an editor of the F-Modeling environment and the user is requested to change the $\boldsymbol{a}$-character or the formula before the code generation. This discovering is based on a special context file attached to the scene $\boldsymbol{a}$-picture and saved in a relational database supporting galleries of $\boldsymbol{a}$-characters. The file includes a minimal distance between nodes of the traversal process and boundary of the 2-D structure. This allows the editor to discover the array-bound errors by the comparison the distance mentioned with a distance of neighborhood available in the formula.

Of course, discovering "run-time errors" before the code generation is possible if necessary pieces of knowledge can be produced and acquired in advance.

Technically, the intelligent aspects of F-modeling environment are based on a collection of the following decision support tools and techniques:

- An open set of $\boldsymbol{a}$-characters (supported by context files, syntax/semantics features, and technical settings) and editors of CyberFrames

- CyberScenes and editors of CyberFrame series

- $\mathrm{C}++$ template programs of CyberScenes

- Algorithms of automatic checking the compatibility of different views

- Code generation from CyberFilm specifications 
- Galleries and clarity annotations of various types

- Libraries of CyberScenes, CyberFilms, and template programs.

All these technologies are based on some types of data/knowledge acquisition being implemented through extensions of the galleries and ontology type libraries. Editors, checkers, and the code generator (all together) can be considered as Unified Knowledge Management system designed to take into account such extensions. The acquisition is not performed through independent adding a piece of information resources (an $\boldsymbol{a}$-character, a CyberFrame or a CyberFilm) into a gallery or a library with consequent attaching links (associations) to other pieces, but through simultaneous adding a cluster of the pieces oriented to a few different galleries and libraries. This means that from very beginning intra-cluster links are designed to efficiently perform some predefine operations and tasks based on some awareness of application model features. In general, F-modeling environment is developed as Active Knowledge (Electronic) Book where users can quickly find necessary information resource, understand it and immediately use (re-use) for new goals. Now the data/knowledge acquisition for this book is performed by the environment developers. End users can also make some contributions but only under special refereeing control.

Items of the above mentioned libraries usually include a number of views presenting a number of different features through multimedia forms. Between different features inside one item and between different items there are a variety of links and associations that support users' decisions on creating their models and for specifying corresponding algorithms, and for automatic synthesis of programs from the CyberFilm specification. The libraries can be considered as ontologies because of their formal basis for searching items, items' associations, necessary features and other actions related to automatic synthesis of programs and intelligent support of user' activities. Usually, each library item obtains double ID to encode its features and a URL-like address. Encoding is based on classification of two types related to "what" is the item from external points of view and "how" is it constructed internally. For example, for 
a whole algorithm, a set of features of input/output data and their (allowed) relations represent "what" code and a set of space structures, traversal schemes on the structures and representative formulas represent "how" code. The search of items in relational database (saving the items) is performed on a combination of features which selection is organized by corresponding editor/browser subsystems.

The interface panels of these subsystems help the users in making decision on which links to go and provide some images of model (information) subspaces and library item associations. This feature and the very-high level of $\boldsymbol{a}$-characters and language constructs decrease chances for the users to be cognitively overloaded and disoriented and empower their cognition through the experience acquired in the libraries. The environment is a mental and computational system that guides, supports and enhances the thinking processes of the users. In addition, it mediates the learning activities that stimulate the cognitive processes and engage learners in creating knowledge that represent their understanding and conception of the information. Some role in stimulating the cognitive processes is also played by removing an essential part of conventional syntactic complexity: the punctuation (such as semicolons and braces), variables for index expressions, applying if-then-else and loop constructs, remembering the names of operations, the order of parameters, misleading terminology, etc.

\section{Scenario of programming in *AIDA}

F-modeling environment is a web-based application providing rich opportunities for creating information resources in *AIDA. It can be installed as a cloud-type service accessed by users via special interface panels on a web browser. Any web browser can be used for presenting these interface panels, however, because of imperfectness in compatibility of some types of file formats, Google Chrome is preferable. When F-modeling environment is launched, the user should go through a conventional login process. After that a starting panel of 
Main view editor will appear.

This panel includes a CyberFrame canvas, a menu bar for creating CyberFrames, searching $\boldsymbol{a}$-pictures (representing $\boldsymbol{a}$-characters) and saving items in libraries. It also includes tool bars to activate various sub-panels for manipulating and updating the canvas contents. In addition, the editor supports attaching annotations to single $\boldsymbol{a}$-pictures and/or to whole CyberFrames. There is a balloon toggle for turning on/off a mode of annotations providing some explanation of the menu bar functions. To create an *AIDA program, there are two ways:

- Searching for an appropriate program in the environment libraries (database) or in personal libraries and editing it according to user's goals, or

- Developing a program "from scratch" by "typing" $\boldsymbol{a}$-characters in rows of Main view CyberFrames.

"Typing" $\boldsymbol{a}$-characters in rows of a new CyberFrame (which is introduced by clicking micro-icon in the menu bar) is started with declarations of space data structures, possible substructures and variables attached to them. The structures and substructures should be accompanied by values of size parameters, and names (identifiers) of the variables should follow the standard type declarations of integer, real, etc. types. "Typing" these $\boldsymbol{a}$-characters is performed by inserting operations above mentioned, that is by opening corresponding sub-panels (representing structures/substructures, data types, etc.). In addition, a conventional typing of values of size parameters (requested by the editor), variable identifiers and possibly structure identifiers is performed. Boxes including all variables attached to the same structure are created automatically.

After the declarations, "typing" $\boldsymbol{a}$-characters in rows representing $\boldsymbol{a}$-scenes is performed. For each $\boldsymbol{a}$-scene, first an $\boldsymbol{a}$-character (from an $\boldsymbol{a}$-scene sub-panel) is selected and inserted at the beginning of a row. This action leads automatically to appearance of micro-icons (cycles or squares) related to operations which should be specified for the $\boldsymbol{a}$-scene, and boxes where the operations should be 
defined. Assignment statements with left and right sides (separated by a special arrow) are usually used for defining the cycle-related operations (also called terminal operations, or just formulas), and other $\boldsymbol{a}$-scenes are used for defining square-related operations (also called non-terminal operations). Examples and details of *AIDA program preparation are presented in *AIDA and F-modeling tutorials available at [38].

In general, the *AIDA program preparation, compiling and running are similar to corresponding operations with conventional programs. The differences are related to manipulations with super-characters (not only with conventional characters) through calls of special sub-panels, as well as to human-system interactions initiated by the editor of F-Modeling environment. These interactions are to clarify meaning of user's intentions (syntax/semantics correctness of the picture-based expressions) and possible features of application algorithms. Requests for compiling and running are implemented by creating intermediate $\mathrm{C}++$ codes.

\section{Case studies and usability tests}

Continuing development of the galleries and libraries (by considering new algorithms and forms of their representations) and the use of them in reallife educational processes are considered as our permanent case studies and usability tests for this new environment. Within these studies and tests, not only understanding algorithms and developing programming skill are analyzed, but also cognitive adaptation of the users within their works on application models is considered. Such analysis and consideration are our guidelines for the environment development. Specific results of the usability tests related to the picture-based program compactness, the program construct comprehension, the library item evaluation, and quality of the code generation can be found in [37][30] [34][32], respectively.

The effectiveness of the approach is also evaluated by a variety of algorithms checked for representing in the picture-based forms. Table 1 presents a current 
list of 57 such algorithms. Practically, this variety is permanently increased because the environment is based on an open set of super-characters allowing the extension of the algorithmic pictures, as well as on adding and editing the embedded clarity annotations. A version of *AIDA web site and related tutorial materials are now available at [38] and we regularly obtain comments and recommendations on enhancing the approach.

Our recent work on 2-year project for JNES (Japan Nuclear Energy Safety organization) can also be considered as a usability test. Algorithms defined by JNES were presented in *AIDA and evaluated by external examiners. The positive evaluation has been a basis for continuing the contract. Here we would like to show results of one of such test related to a $2 \mathrm{D}$ thermal flow algorithm. Within this test, the compactness of the *AIDA constructions was compared with the compactness of corresponding FORTRAN codes and the comprehension of both representations was also evaluated (the Main view of the *AIDA program is available at [7]). All twelve examiners evaluated the comprehension of the *AIDA program as "much higher." A basis for such evaluation can be seen in results of the statistic comparisons shown in Table 2. This includes numbers of pages, lines, variables declared, assignment statements, symbols in index expressions, as well as numbers of loops and branch statements.

In general, based on our current experience, the advantages of *AIDA programs are increased with growing sizes of application algorithms.

\section{Conclusion}

A brief overview of programming in algorithmic pictures has been presented and a number of techniques that are used to enhance perception, comprehension and cognition of people working on developing computational application models and corresponding algorithms have been provided. These techniques are based on special super-characters and their compositions within compound constructs, on the use of background, foreground and auxiliary images (including text, if necessary), and on representing scalable forms of model spaces and 
Table 1: A list of developed algorithms in the pictures

\begin{tabular}{|c|c|}
\hline Categories & Algorithms/Problems \\
\hline \multirow[t]{3}{*}{ Sorting } & . Bubble Sort \\
\hline & . Selection Sort \\
\hline & . Merge Sort \\
\hline \multirow{5}{*}{ Operations on Heaps } & . Up-Heap and Down Heap Operations \\
\hline & . Top-Down Heap Construction \\
\hline & . Bottom-Up Heap Construction \\
\hline & . Priority Queue \\
\hline & . Heap Sort \\
\hline \multirow[t]{4}{*}{ Tree Traversals } & - Pre-order Traversal \\
\hline & . In-order Traversal \\
\hline & - Level-order Traversal \\
\hline & - Post-order Traversal \\
\hline \multirow{6}{*}{ Graph Traversals } & . Depth First Search Tree \\
\hline & . Finding Articulation Points \\
\hline & . Finding Bridges \\
\hline & - Topological Sort \\
\hline & . Breadth First Search for Finding Shortest Path \\
\hline & Finding Strongly Connected Components \\
\hline \multirow[t]{2}{*}{ Minimum Spanning Tree } & · Prim's Algorithm \\
\hline & . Kruskal's Algorithm \\
\hline \multirow[t]{3}{*}{ Single Source Shortest Path } & · Bellman-Ford Algorithm \\
\hline & . Dijkstra's Algorithm \\
\hline & - Priority First Search \\
\hline \multirow[t]{3}{*}{ Network Flow } & . Edmonds-Karp Algorithm \\
\hline & · Dinic's Algorithm \\
\hline & . Push Relabel Algorithm \\
\hline \multirow{8}{*}{ Dynamic Programming } & . Fibonacci Number \\
\hline & Binomial Coefficients \\
\hline & . Matrix Chain Multiplication \\
\hline & - Longest Increasing Subsequence \\
\hline & . Longest Common Subsequence \\
\hline & · Knapsack Problem \\
\hline & . Maximum Sum Problem \\
\hline & . Largest Square Problem \\
\hline Compression & · Huffman Encoding \\
\hline Search & . Binary Search \\
\hline \multirow[t]{12}{*}{ Numerical Analysis and Simulations } & - Gauss Elimination Method \\
\hline & - Gauss Jordan Method \\
\hline & · LU Decomposition \\
\hline & - Matrix Multiplication \\
\hline & - Sparse Matrix-vector Multiplication \\
\hline & - Dirichlet Problem for Laplace's Qquation \\
\hline & . Thermal Conduction Problem \\
\hline & - Least-squares Optimization Method \\
\hline & - Multigrid Method for Solving Partial Diferential Equations \\
\hline & · Particle-In-Cell Algorithms \\
\hline & · Fluid Dynamics Problem \\
\hline & - Monte Carlo Method \\
\hline \multirow[t]{2}{*}{ String Matching } & . Brute Force Algorithm \\
\hline & . Knuth-Morris-Plat Algorithm \\
\hline Computational Geometry & . Convex Hull Algorithms \\
\hline Multi-agent Simulation & . Boid Model Simulation \\
\hline \multirow[t]{2}{*}{ Image Processing } & . Template Matching \\
\hline & . JPEG Compression \\
\hline \multirow[t]{3}{*}{ Cellular Automata-like Algorithms } & Thinning Images \\
\hline & Routing Traffic \\
\hline & - Forest Fire Simulation \\
\hline
\end{tabular}


Table 2: Comparative statistics of a *AIDA program and its equivalent in FORTRAN

\begin{tabular}{lll}
\hline Features to compare & *AIDA & FORTRAN \\
\hline The number of pages & 1 & 8 \\
The number of lines & 22 & 323 \\
The number of variables declared & 50 & 92 \\
The number of assignment statements (=) & 41 & 89 \\
The number of symbols in index expressions (in [ ]) & 49 & 492 \\
The number of loops (do, for, and while statements) & 2 & 48 \\
The number of branch constructs (if-statements) & 2 & 15 \\
\hline
\end{tabular}

attributes declarations, features of algorithmic steps, and multiple views of algorithm as a whole, as well as on special acquisition of them in ontology type galleries and libraries. In this environment, the confidence of users in making decisions is based on access to previous decisions of others, on quick perception and understanding these decisions and on their transformations leading to new models and algorithms.

The interface panels of the environment help the users for making decision and decreases chances for them to be cognitively overloaded and disoriented. They guide, support and enhance the thinking processes of the users and block their error actions. An essential aspect of the environment is various opportunities for users to present important features of their application models, including features of possible input parameters and corresponding simulation algorithms. Very often, such features do not have a direct influence on the code generation, but very useful for readability and understandability of *AIDA programs. Immediate access to these features simplifies visual debugging, modifications and maintenance of the programs, as well as inspections of external examiners. This gives also new opportunities to application programmers for focusing on their models and simulation experiments rather than on problems of the efficient implementation.

\section{Acknowledgements}

We are grateful to JNES (Japan Nuclear Energy Safety organization) for financial support of the *AIDA project and for participation in analysis of its results. This work was supported by JST (Japan Science and Technology Agency) 
Center for Revitalization Promotion.

\section{References}

[1] Beckers, F., 2009. Pictures worth a thousand tiles, a geometrical programming language for self-assembly, Theoretical Computer Science 410(16), 1495-1515.

[2] Bernstein, P.A., Haas, L.M., 2008. Information integration in the enterprise, Communications of ACM 51(9), 72-79.

[3] Booch, G., 2011. Draw me a picture, IEEE Software 28(1), 6-7.

[4] Carroll, J., 2002. Human-Computer Interaction in the New Millennium, AddisonWesley.

[5] Chaudhuri, S., Dayal, U., Ganti, V., 2001. Database technology for decision support systems, IEEE Computer 34(12), 48-55.

[6] Chaudhuri, S., Dayal, U., Narasayya, V., 2011. An Overview of Business Intelligence Technology, Communications of the ACM 54(8), 88-98.

[7] Colored figures of the paper: http://aida.u-aizu.ac.jp/aida/paper_figures.jsp

[8] Danielsson, H., Jonsson, B., 2001. Pictures as Language, in: Proceedings of the International Conference on Language and Visualisation, Stockholm, Sweden, November 8-9.

[9] Detienne, F., Bott, F., 2002. Software design - cognitive aspects, Springer.

[10] Engelbrech, A.P., 2002. Computational Intelligence: An Introduction, Wiley.

[11] Fensel, D., 2001. Ontologies: A Silver Bullet for Knowledge Management and Electronic Commerce, Springer.

[12] Kindborg, M., McGee, K., 2007. Visual programming with analogical representations: Inspirations from a semiotic analysis of comics, Journal of Visual Languages and Computing 18(2), 99-125.

[13] Klein, G., 1999. Sources of Power: How People Make Decisions, MIT Press.

[14] Klein, G., 2004. The Power of Intuition: How to Use Your Gut Feelings to Make Decisions at Work, Broadway business.

[15] Kleppe, A., Warmer, J., Bast, W., 2003. MDA Explained: The Model Driven Architecture: Practice and Promise, Addison-Wesley.

[16] Larkin, J.H., Simon, H.A., 1995. Why a Diagram Is (Sometimes) Worth Ten Thousand Words, in: Glasgow, J., Marayanan, M.H., Chandrasekaran, B. (Eds.), Diagrammatic Reasoning: Cognitive and Computational Perspectives, AAAI Press/MIT Press, pp. 69-109.

[17] Ma, J., Huang, R., Kawashima, T., Apduhan, B.O., 2011. Spacelog Data Acquisition, Management and Applications in a Gateway-based Smart Space, Journal of Information Science and Engineering 27(1), 35-50. 
[18] Ma, J., Wen, J., Huang, R., Huang, B., 2011. Cyber-Individual Meets Brain Informatics, IEEE Intelligent Systems 26(5), 30-37.

[19] Mellor, S.J., Balcer, M.J., 2022. A Foundation for Model-Driven Architecture, Addison-Wesley.

[20] Mirenkov, N., Vazhenin, A., Yoshioka, R., Ebihara, T., Hirotomi, T., Mirenkova, T., 2001. Self-explanatory components: a new programming paradigm, International Journal of Software Engineering and Knowledge Engineering 11(1), 5-36.

[21] Modeling Language: http://modeling-languages.com/

[22] Modha, D.S., Ananthanarayanan, R., Esser, S.K., Ndirango, A., Sherbondy, A.J., Singh, R., 2001. Cognitive Computing, Communications of the ACM 54(8), 62-71.

[23] Bottoni,P., Civica, R., Levialdi, S., Orso, L., Panizzi, E., Trinchese, R., 2004. MADCOW: a multimedia digital annotation system, in: Proceedings of the working conference on Advanced visual interfaces, pp.55-62.

[24] Bottoni, P., Cotroneo, A., Cuomo, M., Levialdi, S., Panizzi, E., Passavanti, M., Trinchese, R., 2010. Facilitating interaction and retrieval for annotated documents, International Journal of Computational Science and Engineering 5(3/4), 197-206.

[25] Bottoni, P., Parisi-Presicce, F., Taentzer, G., 2003. Coordinated Distributed Diagram Transformation for Software Evolution, Electronic Notes in Theoretical Computer Science, 72(4), 59-70.

[26] Parnas, D., 2011. The Risks of Stopping Too Soon, Communications of the ACM 54(6), 31-33.

[27] Petre, M., 1995. Why Looking Isn't Seeing: Readership Skills and Graphical Programming, Communication of the ACM 38(6), 33-44.

[28] Raistrick, C., Francis, P., Wright, J., Carter, C., Wilkie, I., 2004. Model Driven Architecture with Executable UML, Cambridge University Press.

[29] Rasmussen, J., Pejtersen, A.M., Goodstein, L.P., 1994. Cognitive Systems Engineering, John Wiley \& Sons, Inc.

[30] Roxas, R., Mirenkov, N., 2005, Cyber-Film: A Visual Approach that Facilitates Program Comprehension, International Journal of Software Engineering and Knowledge Engineering 15(6), World Scientific, 941-975.

[31] Shneiderman, B., 2002. Leonardo's laptop: human needs and the new computing technologies, MIT Press.

[32] Vazhenin, D., Mirenkov, N., Vazhenin, A., 2001. Movie-based representation of reduction operations in numerical computing, Journal of Knowledge-Based Systems 24, 977-988.

[33] Watanobe, Y., Mirenkov, N., 2012. AIDA Compiler: a Code Synthesizer from Programs in Pictures, in: Proceedings of the Joint International Conference on Human-Centered Computer Environments, pp.76-83. 
[34] Watanobe, Y., Mirenkov, N., Yoshioka, R., 2009. Algorithm Library based on Algorithmic CyberFilms, Journal of Knowledge-Based Systems 22(3), 195-208.

[35] Watanobe, Y., Mirenkov, N., Yoshioka, R., Monakhov, O., 2008. Filmification of methods: A visual language for graph algorithms, Journal of Visual Languages and Computing 19(1), 123-150.

[36] Yen, N.Y., Shih, T.K., Jin, Q., 2012. LONET: An Interactive Search Network for Intelligent Lecture Path Generation, ACM Transactions on Intelligent Systems and Technology.

[37] Yoshioka, R., Mirenkov, N., 2002. Visual Computing within Environment of Selfexplanatory Components, Soft Computing Journal 7(1), 20-32.

[38] *AIDA web site: http://aida.u-aizu.ac.jp/aida/index.jsp 


\section{Figure captions}

Figure 1: A structure scheme of the Algorithmic CyberFilm format

Figure 2: The Main view of *AIDA program for Example 1

Figure 3: The Main view of *AIDA program for Example 2

Figure 4: The Main view of *AIDA program for the Dirichlet problem

Figure 5: Explanation of the second scene $\boldsymbol{a}$-picture (by a series of traversal CyberFrames)

Figure 6: Explanation of the third scene $\boldsymbol{a}$-picture (by a series of traversal CyberFrames)

Figure 7: Explanation of the variable declaration (by attaching units of measure) and "index expressions" (by displaying the neighborhoods of highlighted nodes)

Figure 8: A set of $\boldsymbol{a}$-characters applying for the space structure declarations and traversal scheme specifications

Figure 9: A set of supportive images applying for embedded clarity annotations

Figure 10: Examples of Algorithmic CyberFrames and Background Images

Figure 11: Other examples of $\boldsymbol{a}$-pictures and background images

Figure 12: A scheme of a multilayer Algorithmic CyberFrame

Figure 13: An example of the array-bound error discovery 


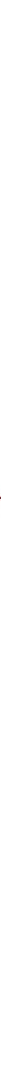
Algorithmic Dynamics
Algorithmic Command
Algorithmic Interface Details Details Details 


\begin{tabular}{|c|c|c|c|}
\hline 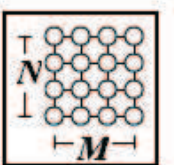 & $\begin{array}{l}N=10 \\
M=1 \\
\text { st }\end{array}$ & 而 & \\
\hline${ }_{c 2}^{c 1}$ & & $r 1: 2, \mathrm{c} 1: 2$ & $r 2: 4, \mathrm{c} 2: 8$ \\
\hline
\end{tabular}

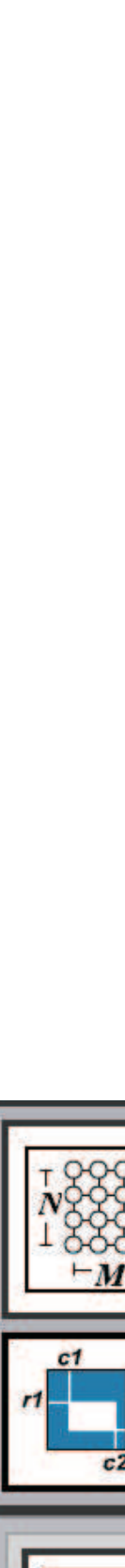

$A[O] \Leftarrow \operatorname{rand}(-5.0,5.0)$

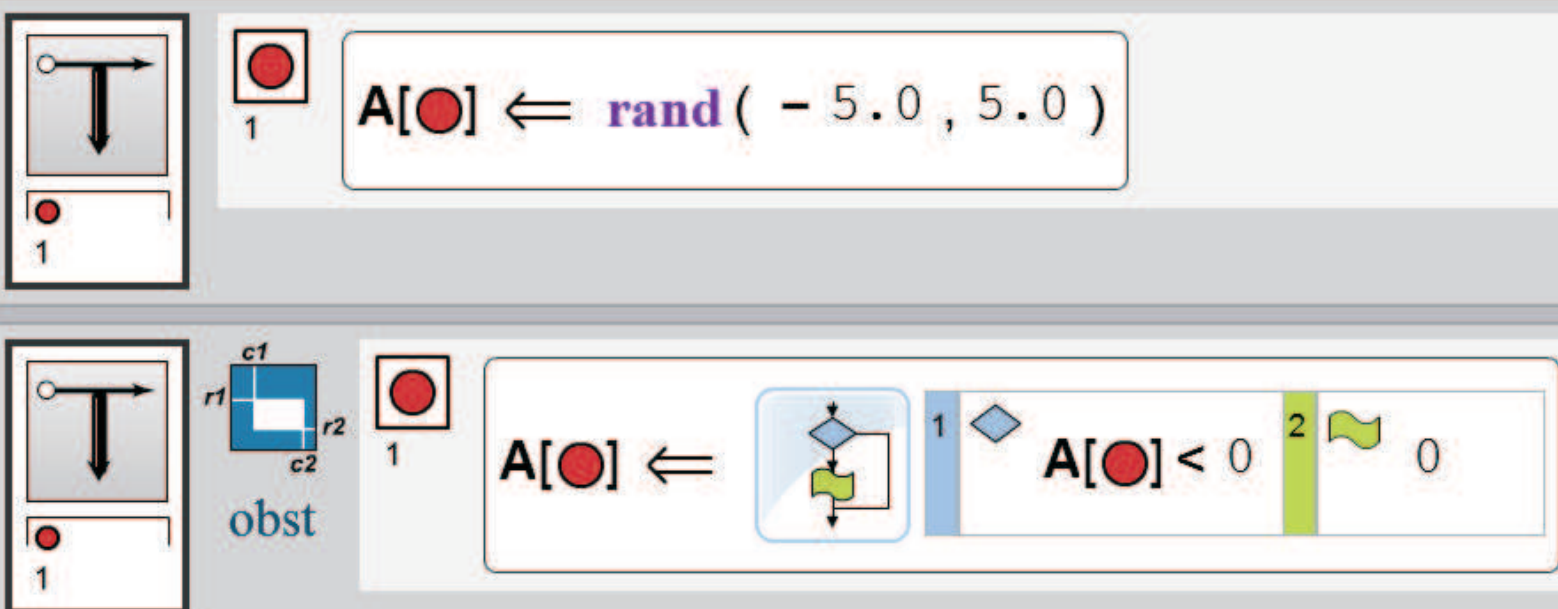

$$
\mathbf{A}[\mathrm{O}] \Leftarrow \sum_{\downarrow}^{\infty} 1 \diamond \mathbf{A}[\mathrm{O}]<0^{2} \cong
$$




\begin{tabular}{|c|c|c|c|c|c|c|}
\hline 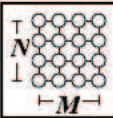 & $\begin{array}{l}N=32 \\
M=32 \\
\text { st1 }\end{array}$ & 而 $\mathrm{A}, \mathbf{B}$ & $\square 1$ & 이 & st2 & 孟 $e \Longleftarrow 0.0025$ \\
\hline
\end{tabular}

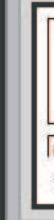

$$
\text { T) }
$$

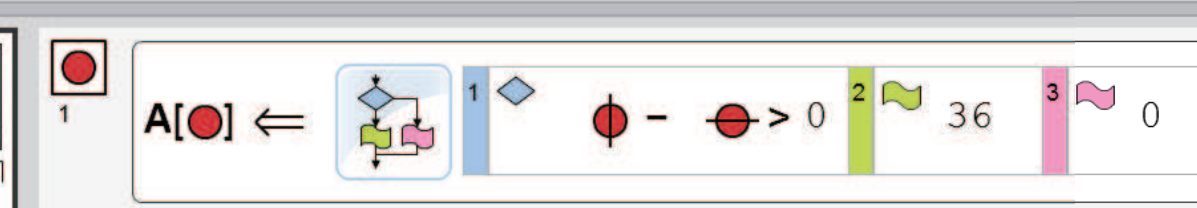

1

$$
8
$$

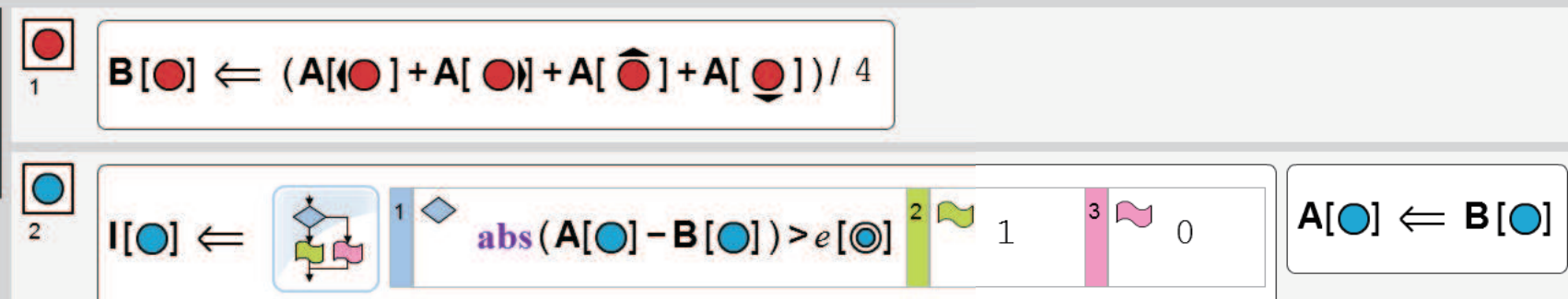

\begin{tabular}{|l|l|c|c|}
\hline$\diamond$ & if & do-frame & else-do-frame \\
\hline & $\bigvee(I[\odot]>0)$ & 1 \\
\end{tabular}

$(1) \in \mathrm{A}[\mathrm{O}]$ 
Figure 5

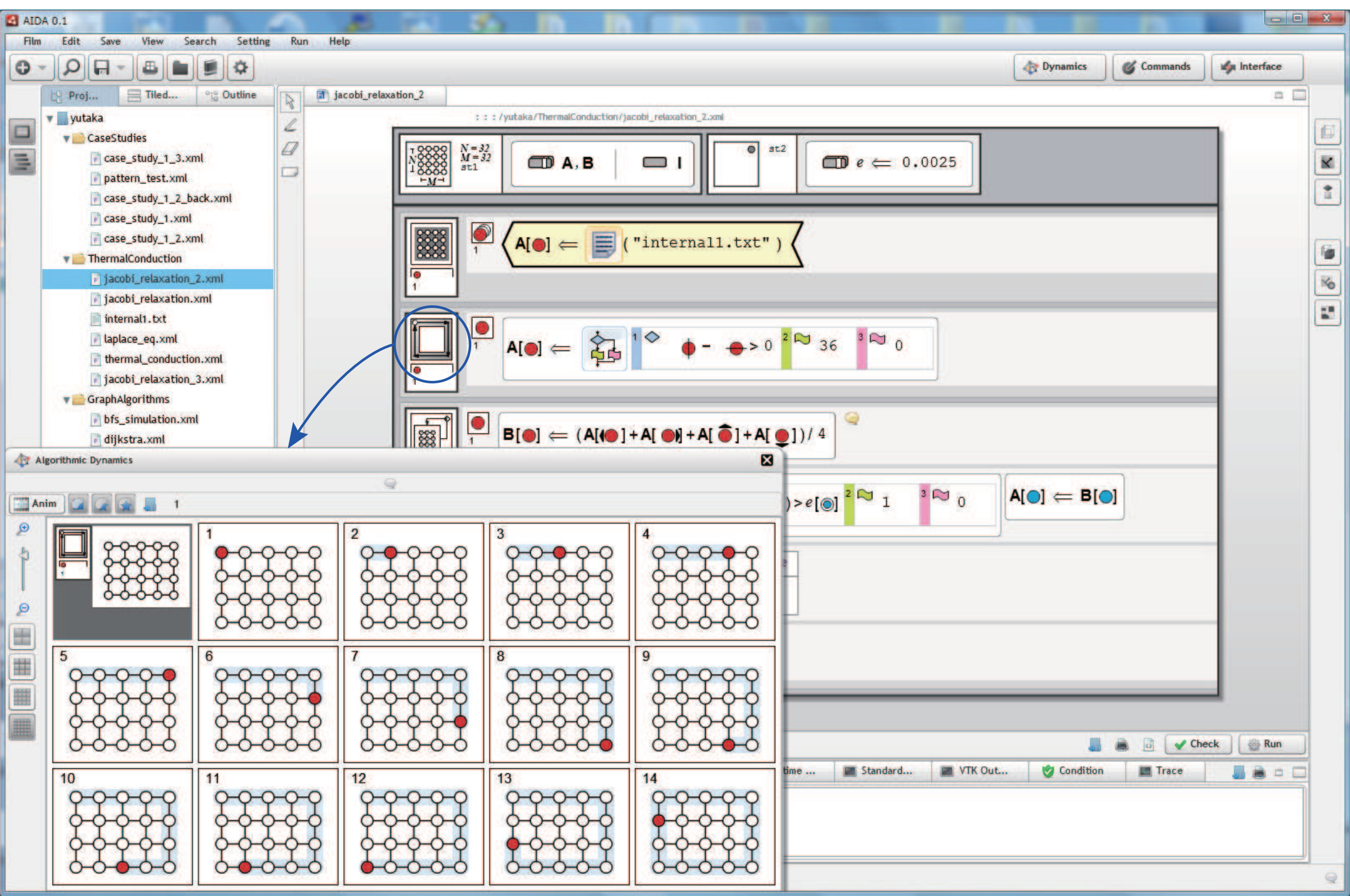


Figure 6

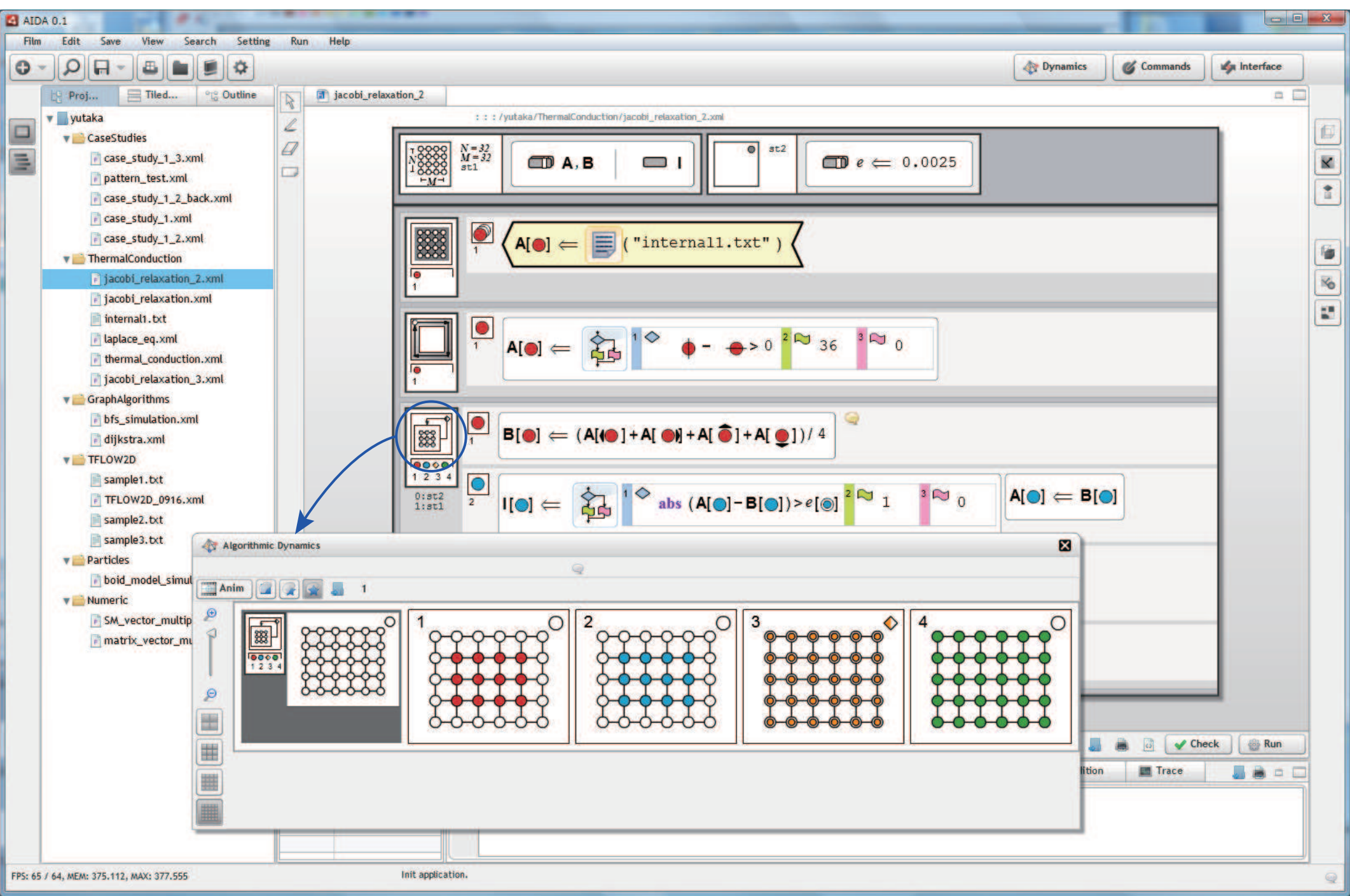




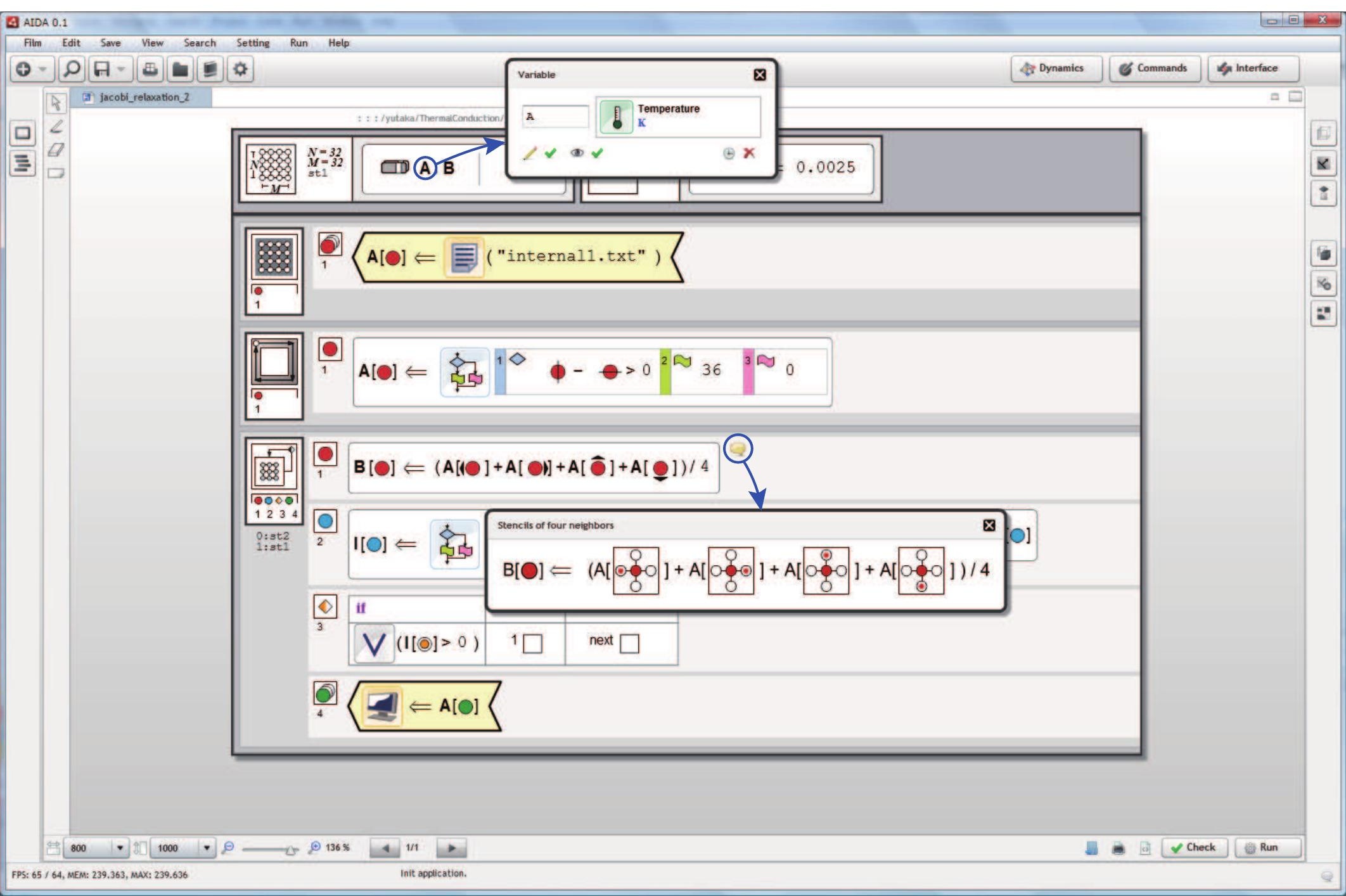



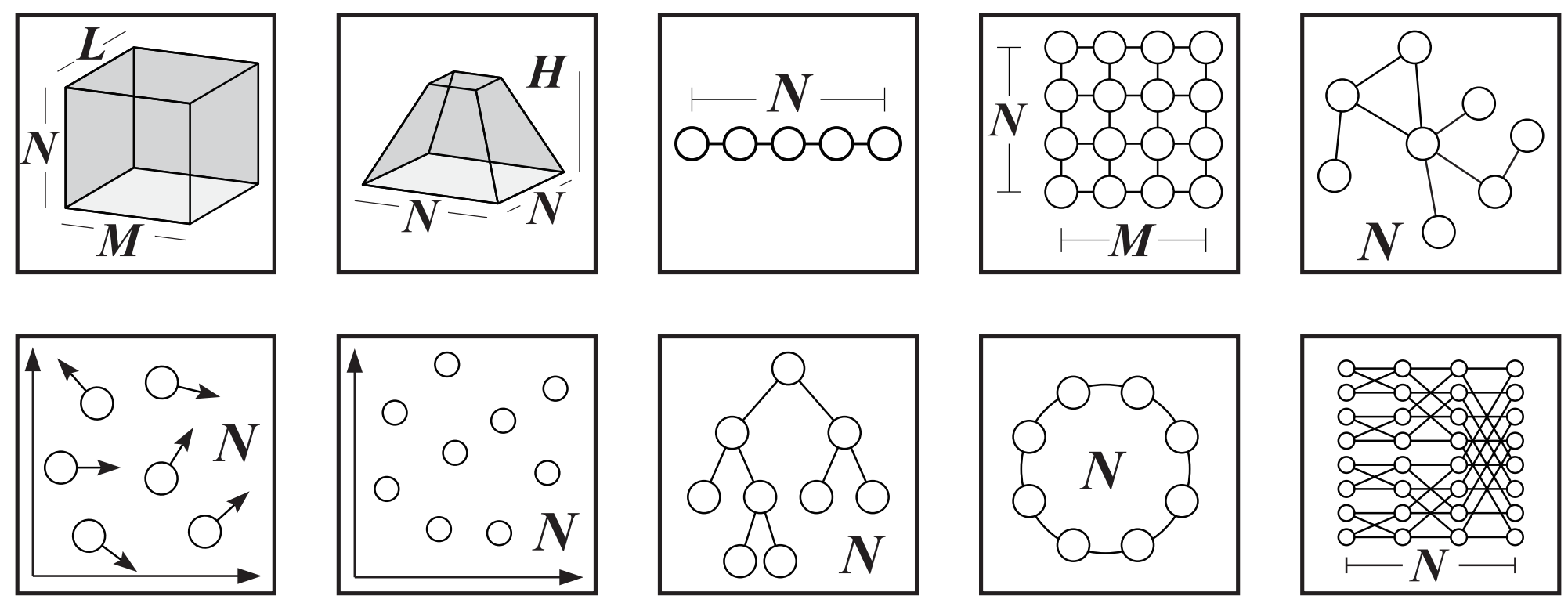

(a)
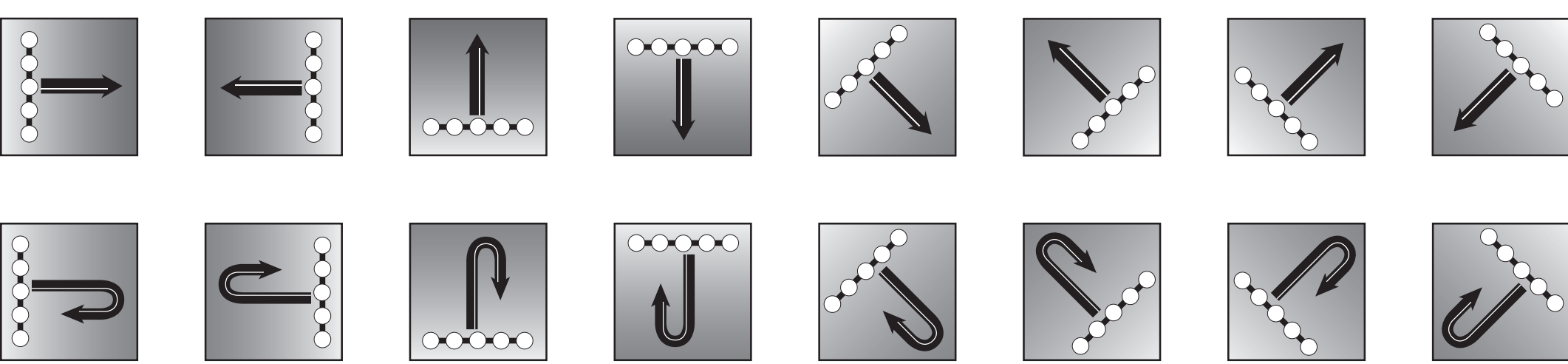

(b) 


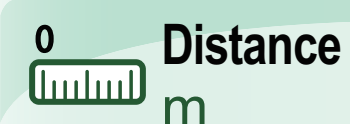

目 Tempreture

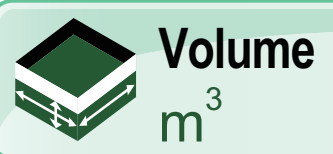

\section{(}

() Energy

$J$

\section{ㄱ) Acceleration $\mathrm{m} / \mathrm{s}^{2}$}

\section{$\dddot{O} \Rightarrow$ Power ... $\mathrm{W}$}

Radioactivity $\mathrm{Bq}$
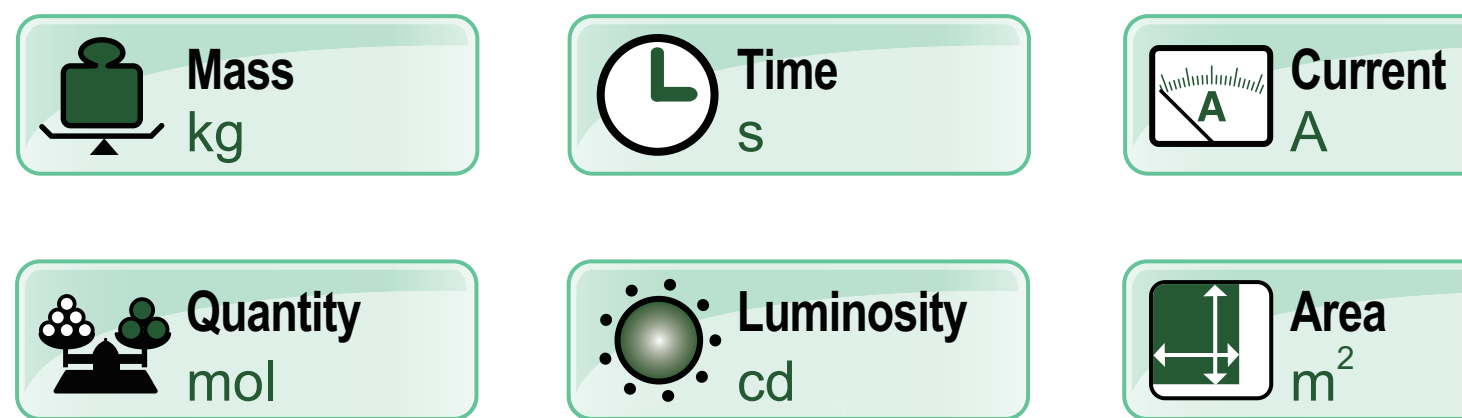

\section{$\rightarrow$ Velocity \\ $\rightarrow \mathrm{m} / \mathrm{s}$}

\section{$\Rightarrow$ Pressure $\rightarrow P a$}

$\theta^{A}$ Angle

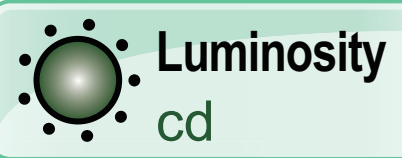

Luminance $\mathrm{cd} / \mathrm{m}^{3}$

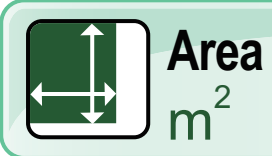

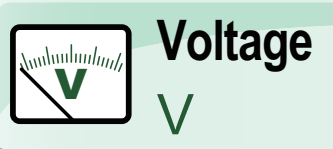

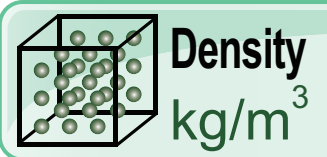

ST. Angular velocity $\mathrm{rad} / \mathrm{s}$

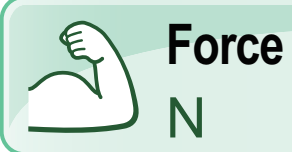

Cis Viscosity

자. Tention

$\mathrm{N} / \mathrm{m}$

$\because::$ Density

$\because: 0 \mathrm{~kg} / \mathrm{m}^{2}$

8. Dose equivalent

Sy
Moment of force $N \cdot m$ 
Figure 10
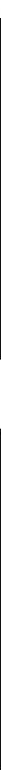

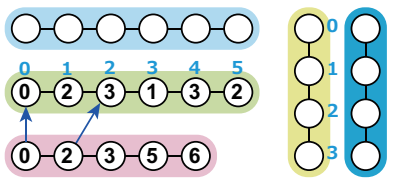




\section{Frame $X$}

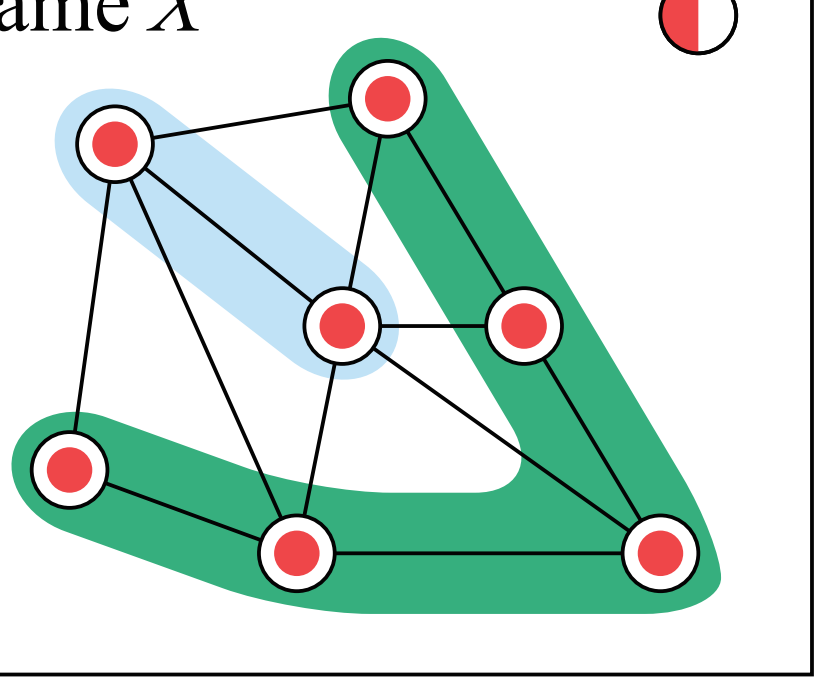

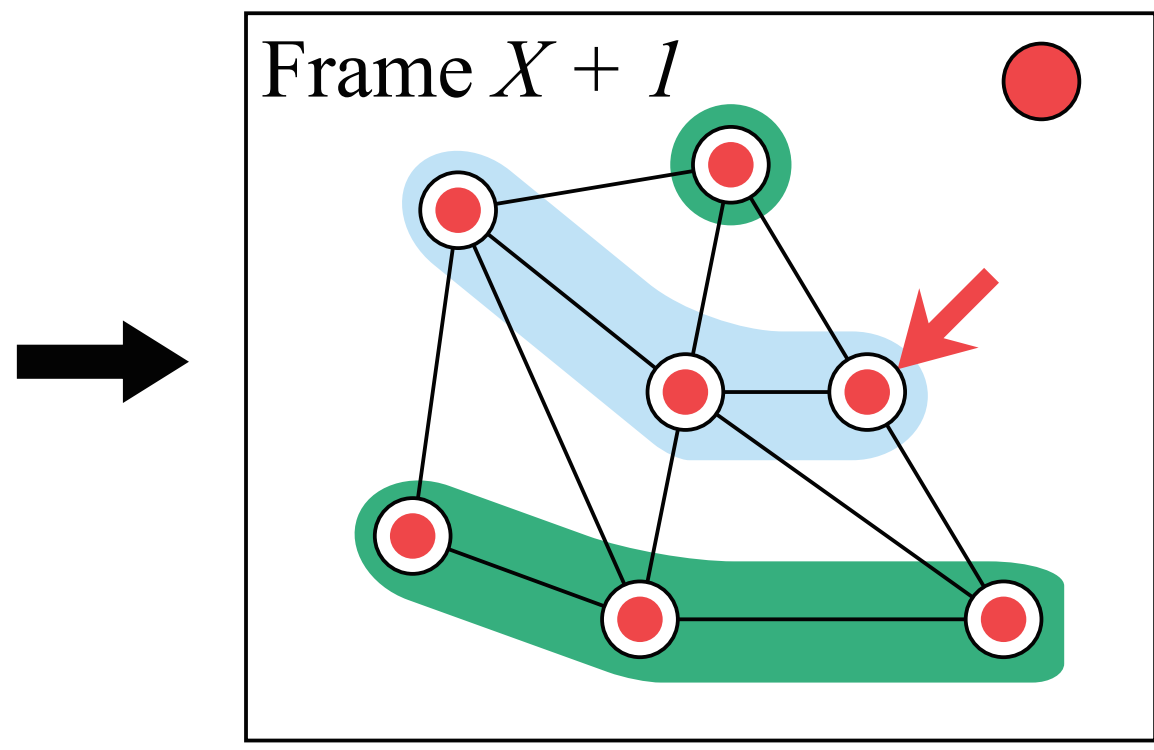

\section{$(1)$ assign $(\bigcirc$ to $\bigcirc$ where edge $[\bigcirc-\infty]$ is minimum}




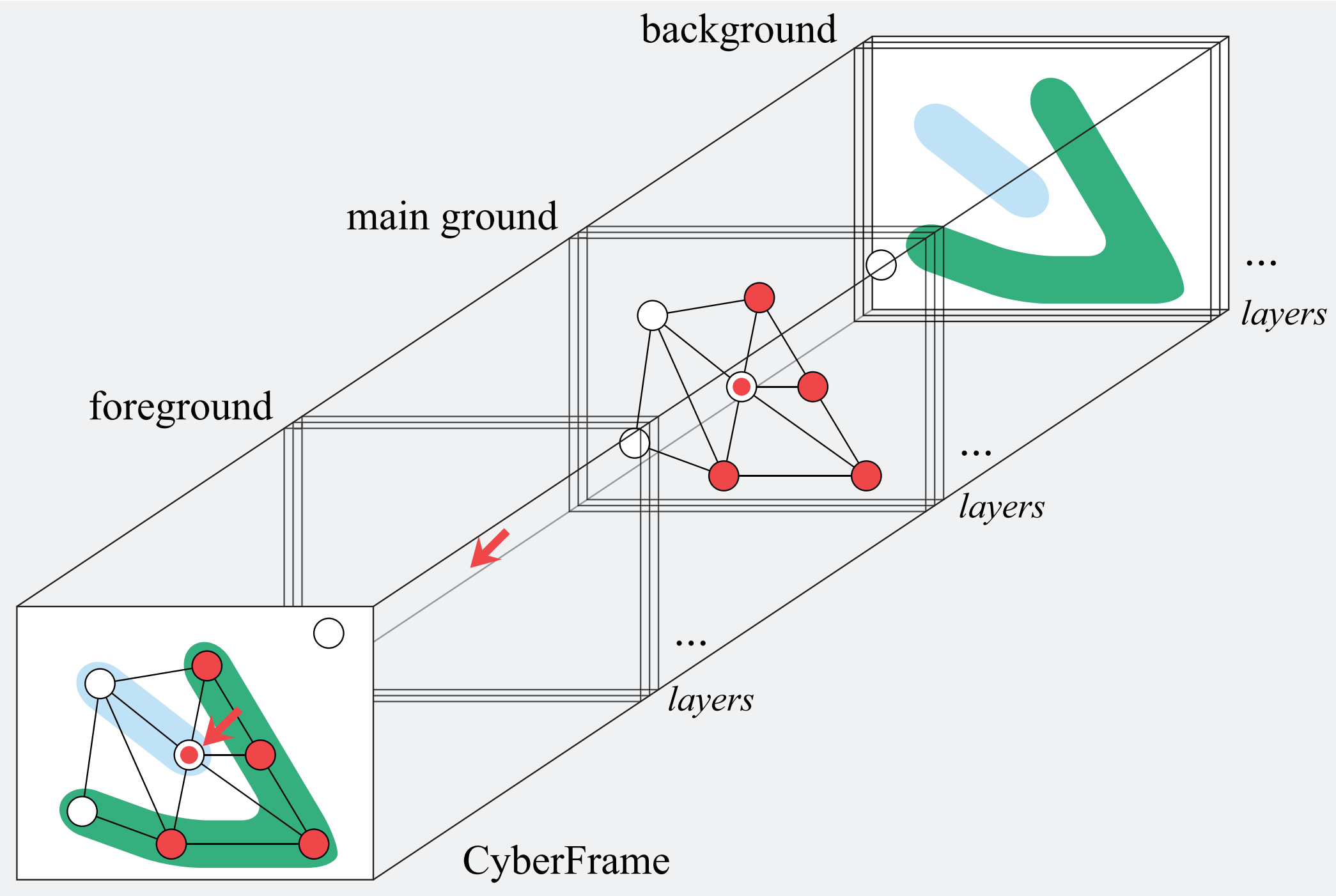




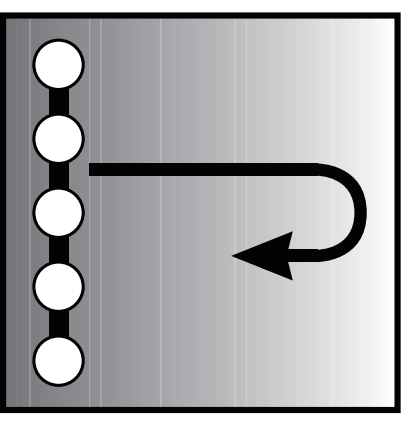

$A[O]=(A[1 O]+A[O /) / 2$
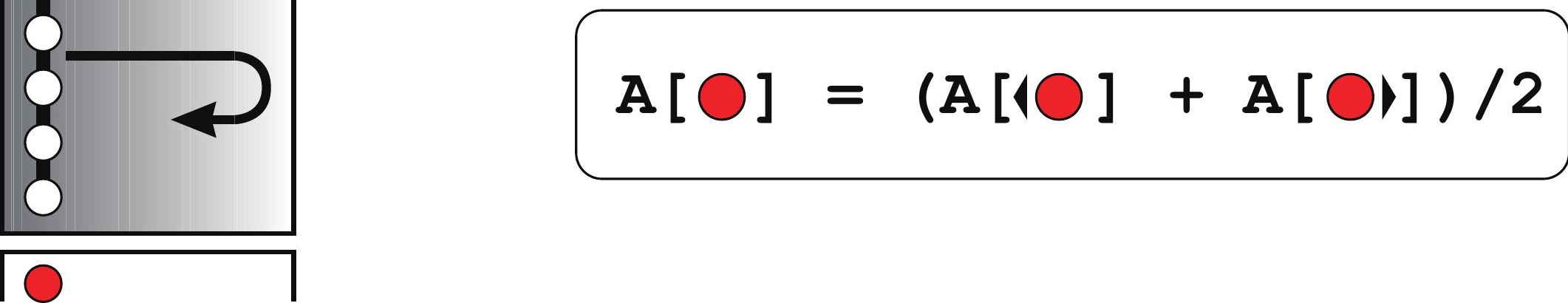

Figure 13 


\section{Yutaka Watanobe}

Dr. Yutaka Watanobe received his M.S. and Ph.D. degree in Computer Science and Engineering from The University of Aizu in 2004 and 2007 respectively. He was a Research Fellow of the Japan Society for the Promotion of Science at The University of Aizu in 2007. He is currently an Associate Professor at The University of Aizu. His research interests includes visual programming, programming in pictures, multimedia programming environments, component (visual) databases, visual query systems using, and filmification of methods as well as high performance computing.

\section{Nikolay Mirenkov}

Professor Nikolay Mirenkov received his PhD and Doctor of Science degree (Habilitation) in Computer Science and Engineering from the Novosibirsk Technical University and the USSR Academy of Sciences in 1972 and 1983, respectively. He came to the University of Aizu (Japan) from the USSR Academy of Sciences (Novosibirsk) in 1993, where he was the head of the supercomputer software department. He was the President of Russian Association "Parallel Computing," an expert of the National Higher Attestation Committee, and a member of Councils awarding higher degrees in research. Since 1993 he has been a professor of the University of Aizu. He was head of the Graduate department of Information systems, head of Computer Software department, dean of the Graduate School of Computer Science and Engineering and Vice President of the University of Aizu. His research interests include programming in pictures, self-explanatory components, human-computer interfaces for people with special needs, visualization, sonification, and filmification of methods and data, as well as parallel programming and high performance computing. 


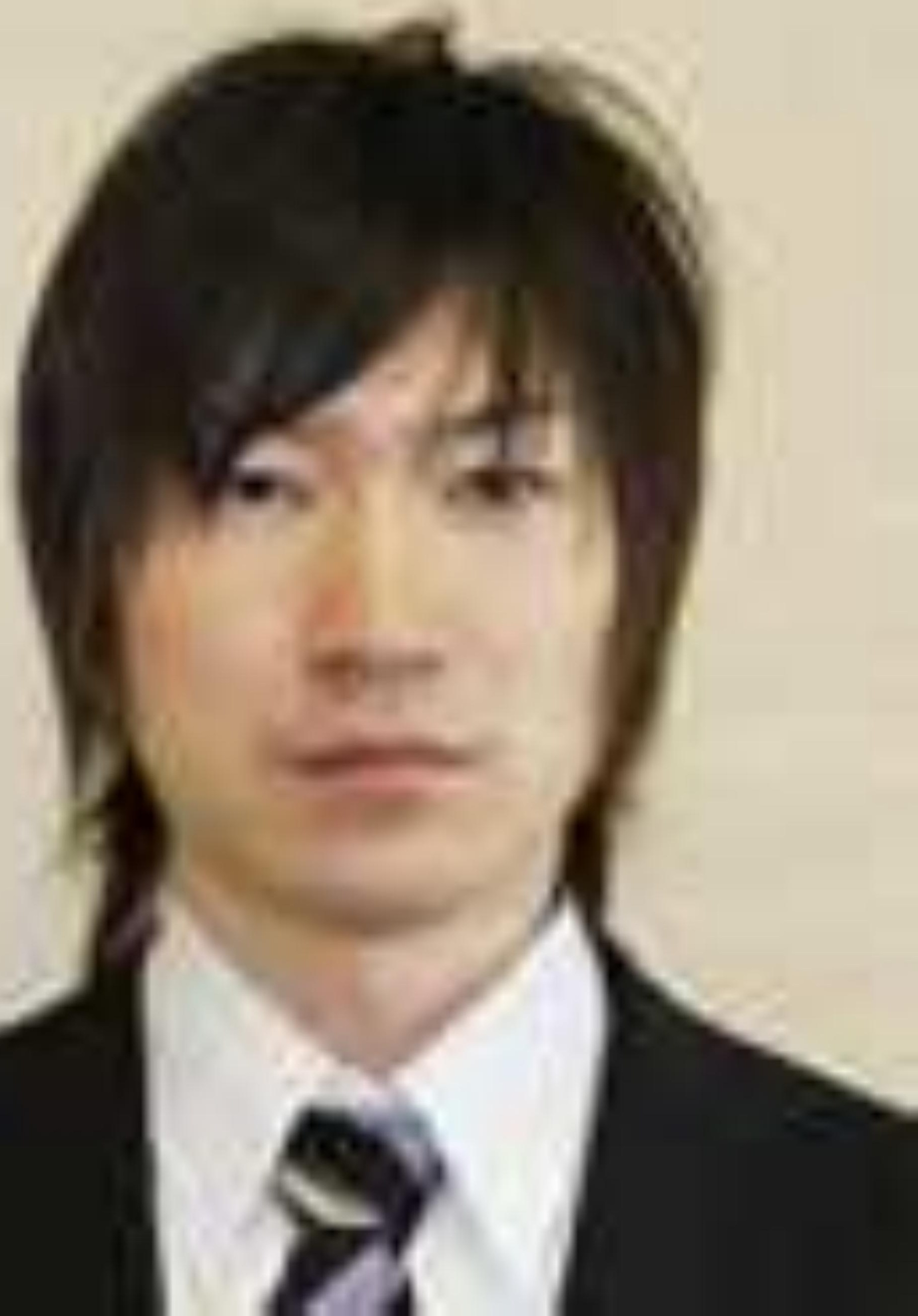




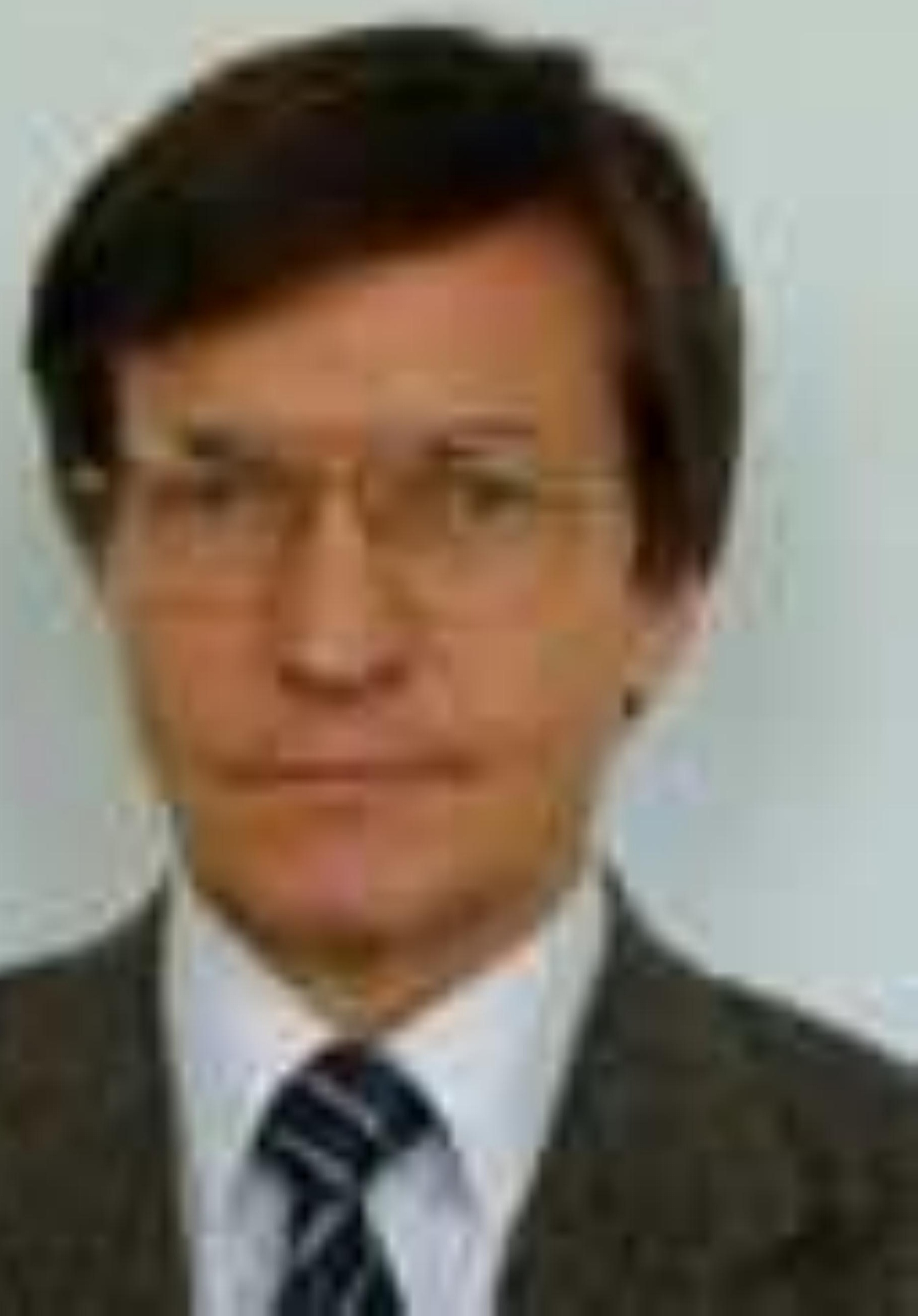

